
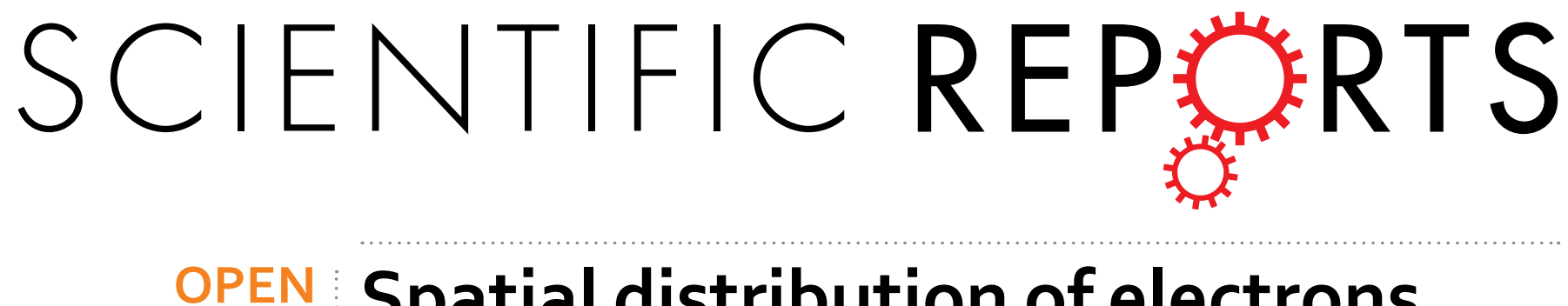

\title{
Spatial distribution of electrons
} near the Fermi level in the metallic $\mathrm{LaB}_{6}$ through accurate $X$-ray charge

Received: 01 August 2016

Accepted: 20 December 2016

Published: 25 January 2017

\section{density study}

Hidetaka Kasai \& Eiji Nishibori

Charge densities of iso-structural metal hexaborides, a transparent metal $\mathrm{LaB}_{6}$ and a semiconductor $\mathrm{BaB}_{6}$, have been determined using the $d>0.22 \AA$ ultra-high resolution synchrotron radiation X-ray diffraction data by a multipole refinement and a maximum entropy method (MEM). The quality of the experimental charge densities was evaluated by comparison with theoretical charge densities. The strong inter-octahedral and relatively weak intra-octahedral boron-boron bonds were observed in the charge densities. A difference of valence charge densities between $\mathrm{LaB}_{6}$ and $\mathrm{BaB}_{6}$ was calculated to reveal a small difference between isostructural metal and semiconductor. The weak electron lobes distributed around the inter $B_{6}$ octahedral bond were observed in the difference density. We found the electron lobes are the conductive $\pi$-electrons in $\mathrm{LaB}_{6}$ from the comparison with the theoretical valence charge density. We successfully observed a spatial distribution of electrons near the Fermi level from the $X$-ray charge density study of the series of iso-structural solids.

Solid state crystalline materials can be classified by their electrical properties, which are insulator, semiconductor, semimetal, and metal. The properties are governed by a behavior of a few electrons per unit cell in the valence band. Exotic physical properties including high temperature superconductor ${ }^{1}$ have often been observed in a narrow region in between two different properties such as insulator and metal. Several exotic properties have never been accompanied with the structural changes. An isostructural series of materials with different electrical properties is one of ideal system to investigate a structure-property relationship and to discover novel properties.

Metal hexaborides $M \mathrm{~B}_{6}$, where $M$ is alkaline earth or rare earth metal, exhibit metallic and semiconductor properties by changing the $M$ ion. The Bardeen-Cooper-Schrieffer (BCS) type superconductivities of $M_{6} \mathrm{~B}_{6}$ were found in $\mathrm{LaB}_{6}$ and $\mathrm{YB}_{6}{ }^{2}$. The metal hexaborides have $\mathrm{a}_{6}$ octahedron in the structure. The $M$ ion is located at the body-center surrounded by the $\mathrm{B}_{6}$ octahedra. The $\mathrm{B}_{6}$ octahedron has 18 valence electrons. Two electrons per $\mathrm{B}_{6}$ octahedron are required to fulfill the bonding orbital of $\mathrm{B}_{6}$. Metal hexaborides with divalent metal ions are considered to be semiconductor and with the trivalent ions are metal from the consideration ${ }^{3}$. Theoretical study suggests that the $\pi$-electron like an anti-bonding orbital of $\mathrm{B}_{6}$ contributes electrical conductivity in the metallic trivalent $M \mathrm{~B}_{6}^{4}$.

$\mathrm{LaB}_{6}$ is widely used as a cathode material due to the electron-emitting properties, the work function ${ }^{5}$, and the long lifetime. $\mathrm{LaB}_{6}$ also has attracted attention as a transparent conductive material ${ }^{6}$. These properties are closely connected to the electronic structure. The electronic structure and the bonding properties in $\mathrm{LaB}_{6}$ have been investigated by the advanced experimental methods such as the de Haas-van Alphen effect ${ }^{7}$, two dimensional angular correlation of the electron positron annihilation radiation ${ }^{8}$ and the electric-field gradient from the ${ }^{11} \mathrm{~B}$ nuclear-magnetic resonance ${ }^{5}$. These results are consistent with theoretical band structure calculated by many researchers in different kinds of methods ${ }^{9-12}$. All the studies provide almost consistent features near the Fermi level. Hossain et al. reported co-existence of ionic, covalent and metallic bonds in the electronic structure of $\mathrm{LaB}_{6}$ system by the pseudo-potential band calculation ${ }^{9}$. They proposed that the co-existence can account for the high efficiency of the electron emission.

Division of Physics, Faculty of Pure and Applied Sciences, Center for Integrated Research in Fundamental Science and Engineering \& Tsukuba Research Center for Interdisciplinary Materials Science, University of Tsukuba. 1-1-1, Tennodai, Tsukuba, Ibaraki, 305-8571, Japan. Correspondence and requests for materials should be addressed to E.N. (email: nishibori.eiji.ga@u.tsukuba.ac.jp) 
Barium atom located adjacent to $\mathrm{La}$ in a periodic table also forms a metal hexaboride $\mathrm{BaB}_{6}$. Alkaline-earth hexaboride has been considered as a simple semiconductor with band gap of several tens $\mathrm{eV}^{13}$. $\mathrm{A}$ band structure of $\mathrm{BaB}_{6}$ has been investigated by a linear muffin-tin orbital atomic-sphere approximation tight-binding (TB-LMTO-ASA) formalism ${ }^{14}$. The electronic structure of $\mathrm{BaB}_{6}$ has also been calculated by a generalized gradient-corrected density functional theory using ultrasoft pseudopotentials and a plane-wave basis ${ }^{14}$. It was found that the band gap at X point linearly depends on the atomic coordinates of boron. They suggested that the parameters easily change the electronic properties from metallic to insulating.

$\mathrm{BaB}_{6}$ can be a good comparative material to investigate a spatial distribution of electrons near the Fermi level in metallic hexaboride, $\mathrm{LaB}_{6}$. Since, $\mathrm{BaB}_{6}$ is isostructural with $\mathrm{LaB}_{6}$ and the difference of total number of electron per unit cell is only one. The valence band for $\mathrm{LaB}_{6}$ has large dispersion with bottom at $\mathrm{X}$ point in the reported band structures ${ }^{9-12}$. The smallest band gap of $\mathrm{BaB}_{6}$ has been found at $\mathrm{X}$ point from the several theoretical calculations ${ }^{13-15}$. Comparison of the electron distribution between $\mathrm{LaB}_{6}$ and $\mathrm{BaB}_{6}$, in principle, should enable us to visualize the spatial distribution of the conductive electrons in $\mathrm{LaB}_{6}$ from the similarities of their band structures.

The charge density distribution of a material is now one of the most accurate observable of experimental investigation in science. We have developed the high quality measurement method of X-ray powder diffraction data at SPring- 8 powder diffraction beamline ${ }^{16}$. We recently achieved to measure diffraction data available to $d$-spacing range $d>0.29 \AA$ for charge density study of $\mathrm{LiCoO}_{2}{ }^{17}$. The diffraction data were successfully analyzed by a maximum entropy method (MEM $)^{18}$ and a multipole refinement ${ }^{19}$. The experimental procedure we developed has been widely applied to accurate charge density studies of materials such as $\alpha$-boron ${ }^{20}, \mathrm{TiO}_{2}{ }^{21}, \mathrm{Al}_{2} \mathrm{O}_{3}{ }^{21}$, $\mathrm{CoSb}_{3}{ }^{22,23}$, silicon ${ }^{24}$ and diamond ${ }^{24,25}$. The technique successfully determined the detailed charge density features which were quantitatively comparable to those determined theoretically ${ }^{17,20-25}$. Recently, the charge density studies from ultra-high resolution synchrotron radiation powder diffractions have been reported using an all-in-vacuum diffractometer installed at PETRA-III ${ }^{26}$. Core electron deformations of diamond ${ }^{26}$, silicon ${ }^{27}$ and cubic boron nitride ${ }^{28}$, and anharmonic thermal vibration of copper ${ }^{29}$ have been observed from powder diffraction data. Several analytical procedures such as Wilson $\operatorname{plot}^{26-29}$, and uncertainty estimation using particle statics ${ }^{27}$ were used in the studies. These techniques are under development since it still depends on materials ${ }^{26-28}$.

In this study, we investigated the charge densities of divalent and trivalent metal hexaborides, semiconducting $\mathrm{BaB}_{6}$ and metallic $\mathrm{LaB}_{6}$, through the ultra-high resolution powder diffraction data. Accurate charge densities were determined by the experimental and analytical procedure using the multiple powder diffraction datasets at SPring- 8 reported by Nishibori et al. ${ }^{24}$ and Svendsen et al. ${ }^{25}$ We achieved the analysis of the data with the highest reciprocal space resolution, $d>0.22 \AA$. A use of relativistic atomic scattering factors was required for analysis of $d<0.25 \AA$ data.

\section{Results}

Rietveld refinements of $\mathrm{LaB}_{6}$ and $\mathrm{BaB}_{6}$ using two datasets, $\mathrm{D} 1$ and $\mathrm{D} 2$ data, were carried out using program synchrotron powder $(\mathrm{SP})^{24}$. Weak peaks from $\mathrm{Al}_{2} \mathrm{O}_{3}$ found in the diffraction data of $\mathrm{BaB}_{6}$ were treated as a second phase in the Rietveld refinement. The reciprocal resolution used in the analysis for $\mathrm{LaB}_{6}$ and $\mathrm{BaB}_{6}$ are corresponding to $d>0.203 \AA$ and $d>0.225 \AA$, respectively. Reliability factors based on weighted profile, $R_{\mathrm{wp}}$, and Bragg intensity, $R_{\mathrm{I}}$, of $\mathrm{LaB}_{6}$ were $2.27 \%$ and $0.97 \%$ for two datasets and those of $\mathrm{BaB}_{6}$ were $2.22 \%$ and $1.22 \%$ for two datasets.

We extracted observed structure factors from the results of Rietveld refinements. The structure factors of completely overlapped reflections were estimated using the calculated structure factors based on the independent atom model in the Rietveld refinement. We performed multipole refinements of the observed structure factors using the program $\mathrm{XD}^{30}$. The calculated structure factors were updated by the multipole refinement. The ratios between structure factors of the completely overlapped reflections were changed by the multipole model. We improved extraction of the observed structure factors by a powder diffraction pattern fitting using the structure factors from the multipole refinement. The function was added to the originally developed program $\mathrm{SP}^{24}$. The iterative procedure of the multipole refinement and powder pattern fitting was conducted twice for $\mathrm{LaB}_{6}$ and three times for $\mathrm{BaB}_{6}$ until the changes of parameters for multipole refinement were converged within standard uncertainty.

The results of the final powder fittings for $\mathrm{LaB}_{6}$ and $\mathrm{BaB}_{6}$ are shown in Fig. $1 \mathrm{a}$ and b, respectively. The $R_{\mathrm{wp}}$ and $R_{\mathrm{I}}$ of the final powder fittings for $\mathrm{LaB}_{6}$ were $6.39 \%$ and $0.56 \%$ for $\mathrm{D} 1,0.71 \%$ and $0.76 \%$ for $\mathrm{D} 2$, and $2.23 \%$ and $0.69 \%$ for two datasets and those of $\mathrm{BaB}_{6}$ were $5.92 \%$ and $0.92 \%$ for $\mathrm{D} 1,0.86 \%$ and $1.20 \%$ for D2, and $2.20 \%$ and $1.10 \%$ for two datasets. The structure factors extracted based on the fittings were used for the charge density studies by the multipole refinement and MEM.

We detected anharmonic thermal vibrations of boron atoms from a residual density of $\mathrm{LaB}_{6}$ during process of the refinement. The parameters of the anharmonic thermal vibrations of boron atoms for $\mathrm{LaB}_{6} \mathrm{were}$ successfully determined in the multipole refinement. The reliability factors, $R$ and $R_{\mathrm{W}}$, on the final multipole refinement were as small as $0.47 \%$ and $0.40 \%$ for $\mathrm{LaB}_{6}$ and $0.57 \%$ and $0.42 \%$ for $\mathrm{BaB}_{6}$. Those values are much smaller than those of the Rietveld refinement.

Table 1 shows the refined multipole populations and $\kappa$ parameters for $\mathrm{LaB}_{6}$ and $\mathrm{BaB}_{6}$. The anharmonic thermal vibrations by Gram-Charlier temperature factor formalism of $\mathrm{B}$ for $\mathrm{LaB}_{6}$ are also listed in Table 1 . The residual density maps of harmonic and anharmonic thermal parameter model are shown in Supplementary Figure S1. The decrease of the residual in antibond direction of $\mathrm{B}$ atom is recognized in the maps. Several parameters relating to the heavy elements, $\mathrm{La}$ and $\mathrm{Ba}$, were individually refined and fixed due to their unstable behavior in the refinement. The $P_{\mathrm{v}}$ parameters of $\mathrm{Ba}$ and $\mathrm{B}$ in $\mathrm{BaB}_{6}$ were individually refined, then the other multipole parameters were refined. The $\kappa$ and $\kappa$ ' of La were fixed to 1.0 since the parameters became physically meaningless negative values by the refinement. The result for $\mathrm{BaB}_{6}$ shows populations of $P_{20}$ and $P_{30}$ mainly contribute to chemical bonding between boron atoms. The other populations are less than $20 \%$ of $P_{20}$ and $P_{30}$ in $\mathrm{BaB}_{6}$. Similar large $P_{20}$ 

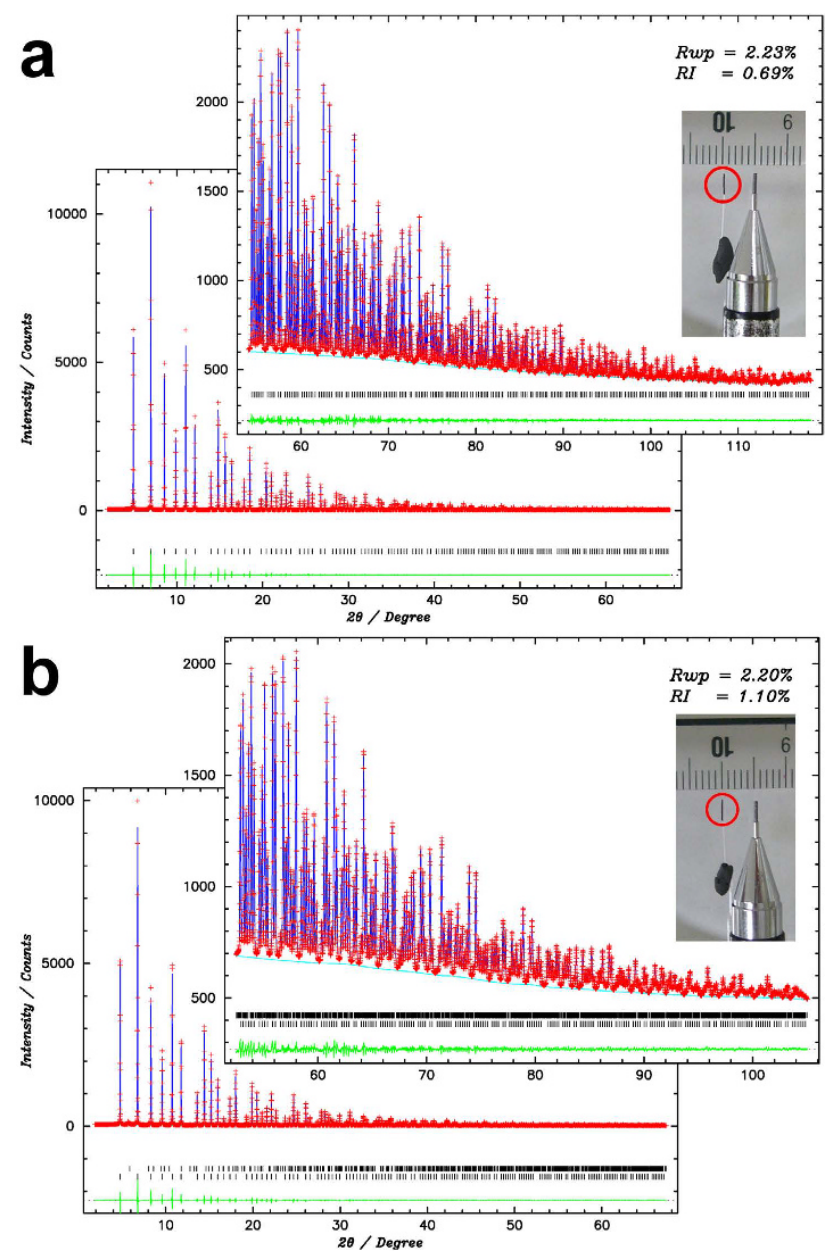

Figure 1. Fitting results of Rietveld refinement. (a) The results of $\mathrm{LaB}_{6}$. (b) $\mathrm{BaB}_{6}$. Insets show the results of high angle D2 data. The photographs of sample are also displayed with scaler. The graphs are drawn by the program using pgplot library (http://www.astro.caltech.edu/ tjp/pgplot/) and edited with Gimp 2.8.14 (https:// www.gimp.org).

\begin{tabular}{|c|c|c|c|}
\hline & & $\mathrm{LaB}_{6}$ & $\mathrm{BaB}_{6}$ \\
\hline \multirow[t]{5}{*}{$M$} & $P_{\mathrm{v}}$ & $0.47(19)$ & $1.4(2)^{*}$ \\
\hline & $P_{40}$ & $0.10(10)$ & $0.37(9)$ \\
\hline & $P_{44+}$ & $0.07(7)$ & $0.27(6)$ \\
\hline & $\kappa$ & 1 & $0.97(6)$ \\
\hline & $\kappa^{\prime}$ & 1 & $0.95(4)$ \\
\hline \multirow[t]{14}{*}{$\mathrm{B}$} & $P_{\mathrm{v}}$ & $3.09(3)$ & $3.10(4)^{*}$ \\
\hline & $P_{10}$ & $0.048(16)$ & $0.015(12)$ \\
\hline & $P_{20}$ & $0.10(2)$ & $0.122(18)$ \\
\hline & $P_{30}$ & $0.17(4)$ & $0.20(3)$ \\
\hline & $P_{40}$ & $0.08(5)$ & $0.00(3)$ \\
\hline & $P_{44+}$ & $0.09(4)$ & $0.04(3)$ \\
\hline & $\kappa$ & $0.99(3)$ & $0.990(5)$ \\
\hline & $\kappa^{\prime}$ & $1.05(2)$ & $1.11(3)$ \\
\hline & C111 & $0.000050(179)$ & \\
\hline & $\mathrm{C} 112=\mathrm{C} 133$ & $-0.000036(87)$ & \\
\hline & D1111 & $0.000061(121)$ & \\
\hline & $\mathrm{D} 2222=\mathrm{D} 3333$ & $-0.000055(98)$ & \\
\hline & $\mathrm{D} 1122=\mathrm{D} 1133$ & $-0.000038(40)$ & \\
\hline & D2233 & $0.000002(66)$ & \\
\hline
\end{tabular}

Table 1. Multipole refinement details. 

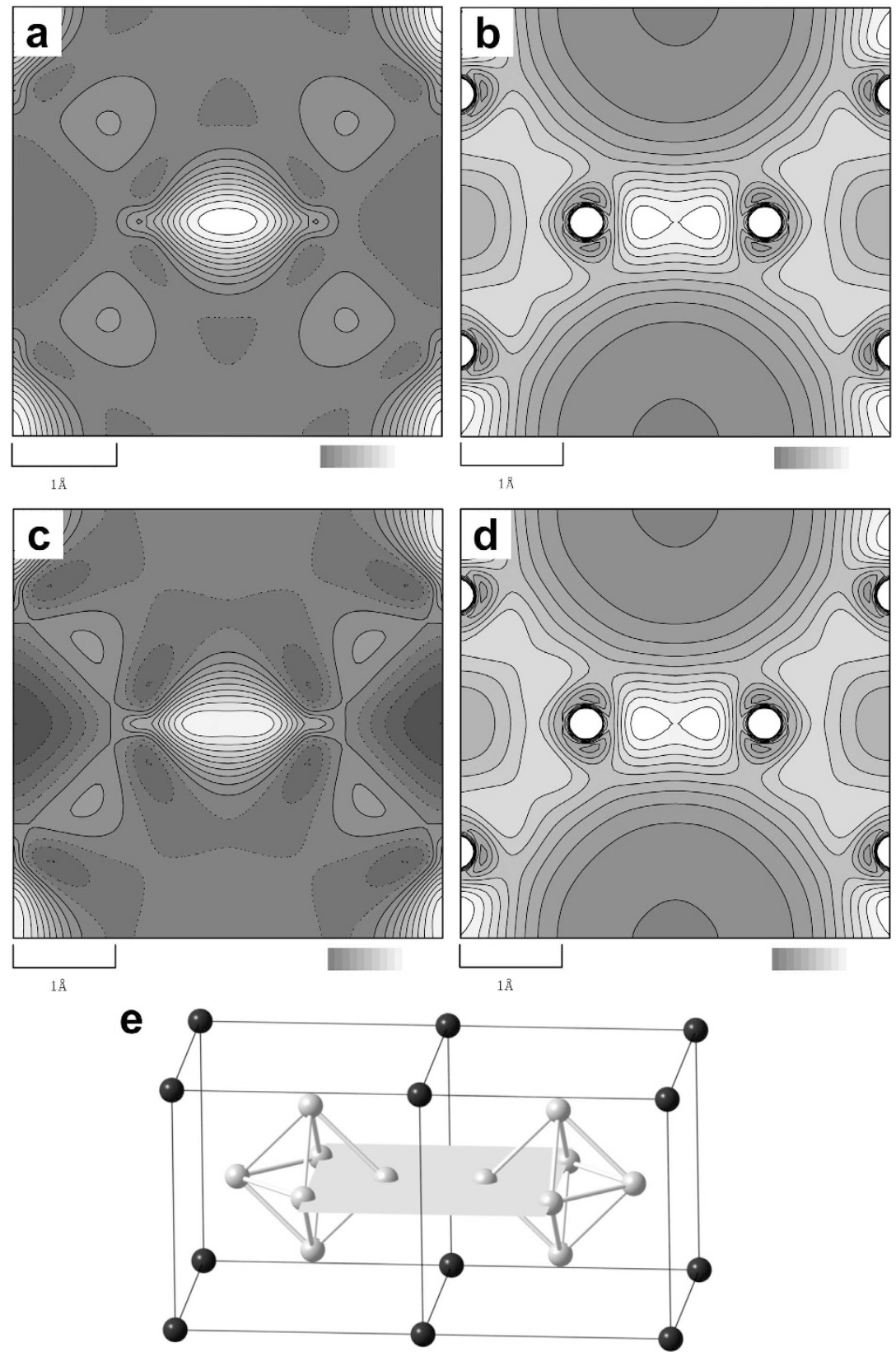

Figure 2. Static deformation density and valence charge density maps, schematic structure by multipole refinement and topological analysis of $\mathrm{LaB}_{6}$ and $\mathrm{BaB}_{6}$ for 020 plane. (a,c) Static deformation maps for (a) $\mathrm{LaB}_{6}$ and $(\mathbf{c}) \mathrm{BaB}_{6}$. Contour lines are drawn from 0.0 to 0.4 with $0.04 e / \AA^{3}$ step. (b,d) Valence charge density maps for $(\mathbf{b}) \mathrm{LaB}_{6}$ and $(\mathbf{d}) \mathrm{BaB}_{6}$. Contour lines are drawn from 0.0 to 1.0 with $0.1 \mathrm{e} / \AA^{3}$ step. Dark gray and white indicate low and high density regions respectively. Color bars are also shown at the bottom of the figures. (e), Schematic figure indicating 020 plane are also shown. The maps are drawn by the program using pgplot library (http://www.astro.caltech.edu/ tjp/pgplot/) and edited with Gimp 2.8.14 (https://www.gimp.org). The schematic figures was drawn with a program Ball\&Stick ${ }^{37}$.

and $P_{30}$ populations are found in $\mathrm{LaB}_{6}$. The populations of $P_{10}, P_{40}$, and $P_{44+}$ for $\mathrm{LaB}_{6}$ are at least twice larger than those of $\mathrm{BaB}_{6}$.

We investigated charge densities of $\mathrm{LaB}_{6}$ and $\mathrm{BaB}_{6}$ by the multipole refinement, the MEM analysis, and the theoretical calculations in the present study. In comparison of $\mathrm{LaB}_{6}$ and $\mathrm{BaB}_{6}$, there was no significant difference in charge densities between $M$ and $\mathrm{B}$. The charge densities between $M$ and $\mathrm{B}$ are shown in the Supplementary Figures S2, S3, and S4. The significant difference between $\mathrm{BaB}_{6}$ and $\mathrm{LaB}_{6}$ was found in the bonding network between boron atoms. The bonding nature of boron atoms is mainly discussed in the following text.

Figure $2 a, b, c$ and $d$ show the static deformation and valence charge density from multipole refinement for $\mathrm{LaB}_{6}$ and $\mathrm{BaB}_{6}$ as contour maps of 020 sections. The charge density section is represented schematically in Fig. $2 \mathrm{e}$. 


\begin{tabular}{|c|c|c|c|c|c|c|}
\hline \multirow[b]{2}{*}{ Bond } & \multirow{2}{*}{$\begin{array}{c}\text { Bond } \\
\text { Path }[\AA]\end{array}$} & \multirow{2}{*}{$\begin{array}{c}\mathrm{LaB}_{6} \\
\rho\left[\mathrm{e}^{-3}\right]\end{array}$} & \multirow[b]{2}{*}{$\nabla^{2} \rho\left[\mathrm{e} \AA^{-5}\right]$} & \multirow{2}{*}{$\begin{array}{l}\text { Bond } \\
\text { Path[§̊] }\end{array}$} & \multirow{2}{*}{$\frac{\mathrm{BaB}_{6}}{\rho\left[\mathrm{e}^{-3}\right]}$} & \multirow[b]{2}{*}{$\nabla^{2} \rho\left[\mathrm{e} \AA^{-5}\right]$} \\
\hline & & & & & & \\
\hline $\mathrm{B}_{6}-\mathrm{B}_{6}$ & 1.657 & 1.12 & -10.07 & 1.746 & 0.99 & -6.77 \\
\hline B-B & 1.763 & 0.71 & -0.52 & 1.777 & 0.73 & -1.52 \\
\hline B-M & 3.049 & 0.14 & 1.51 & 3.135 & 0.12 & 1.33 \\
\hline
\end{tabular}

Table 2. Results of Topological analysis. The esds are not given in XD2006. It is estimated that the esds are the size as the last significant figure.

The six boron atoms are located on the plane. The strong B-B bonds are clearly recognized at the center of the figures. The bond is connecting to $\mathrm{B}_{6}$ octahedra, what we call the $\mathrm{B}_{6}-\mathrm{B}_{6}$ bond. The other charge density overlaps between $\mathrm{B}$ atoms are also recognized at the vicinities edges, what we call the $\mathrm{B}-\mathrm{B}$ bond. There are strong charge density peaks at the $\mathrm{B}_{6}-\mathrm{B}_{6}$ midpoint in Fig. $2 \mathrm{a}$ and $\mathrm{c}$. There are also weak peaks at the $\mathrm{B}-\mathrm{B}$ midpoints. The charge densities at the $\mathrm{B}_{6}-\mathrm{B}_{6}$ bond midpoints of $\mathrm{LaB}_{6}$ are $0.43 \mathrm{e} / \AA^{3}$ and $1.06 \mathrm{e} / \AA^{3}$ in the static deformation and valence densities, respectively. The charge densities at the $\mathrm{B}_{6}-\mathrm{B}_{6}$ bond midpoints of $\mathrm{BaB}_{6}$ are $0.39 e / \AA^{3}$ and $0.99 e / \AA^{3}$ in the static deformation and valence densities, respectively. The charge densities at the $\mathrm{B}-\mathrm{B}$ bond midpoints of $\mathrm{LaB}_{6}$ and $\mathrm{BaB}_{6}$ are $0.08 e / \AA^{3}$ and $0.08 e / \AA^{3}$ in the static deformation densities, respectively.

The topological properties for $\mathrm{LaB}_{6}$ and $\mathrm{BaB}_{6}$ were calculated from the total charge density by the multipole refinements using program TOPOXD ${ }^{30}$. The lengths of bond paths, charge densities and Laplacians at bond critical points (BCPs) are listed in Table 2. The striking difference is found in the bond path length between $\mathrm{LaB}_{6}$ and $\mathrm{BaB}_{6}$. The $\mathrm{B}_{6}-\mathrm{B}_{6}, \mathrm{~B}-\mathrm{B}$ and $\mathrm{B}-M$ lengths of $\mathrm{LaB}_{6}$ are $1.657 \AA, 1.763 \AA$, and $3.049 \AA$ which are $0.089 \AA, 0.014 \AA$, and $0.086 \AA$ shorter than those of $\mathrm{BaB}_{6}$. The $\mathrm{B}_{6}-\mathrm{B}_{6}$ and $\mathrm{B}-M$ distances mainly contribute to the $0.11 \AA$ difference of the lattice constant between 4.149410(2) $\AA$ for $\mathrm{LaB}_{6}$ and 4.258990 (3) $\AA$ for $\mathrm{BaB}_{6}$. Structure parameters are also listed in Supplementary Table S1. The observed structural changes are consistent with previous structural studies ${ }^{14,31}$.

Chemical bonding in second row elements can be simply interpreted by the Laplacians ${ }^{32}$. The Laplacians at the $\mathrm{BCP}$ of the $\mathrm{B}_{6}-\mathrm{B}_{6}$ bonds are $-10.07 e / \AA^{5}$ for $\mathrm{LaB}_{6}$ and $-6.77 e / \AA^{5}$ for $\mathrm{BaB}_{6}$. These are covalent bonding interactions in both the materials. The covalent interaction from the Laplacian of $\mathrm{LaB}_{6}$ is almost half and that of $\mathrm{BaB}_{6}$ is almost one-third of that of diamond, $-21.3 e / \AA^{5}$. The covalency in the $\mathrm{B}_{6}-\mathrm{B}_{6}$ of $\mathrm{LaB}_{6}$ is stronger than that of $\mathrm{BaB}_{6}$. The Laplacian at the $\mathrm{BCP}$ of the $\mathrm{B}-\mathrm{B}$ bond of $\mathrm{BaB}_{6}$ indicates also the covalent bonding interaction, $-1.52 e / \AA^{5}$, and the covalency of $\mathrm{BaB}_{6}$ at this point is stronger than that of $\mathrm{LaB}_{6},-0.52 \mathrm{e} / \AA^{5}$. In other words, the boron atom in $\mathrm{LaB}_{6}$ has $\mathrm{B}-\mathrm{B}$ dimer-like feature than that of $\mathrm{BaB}_{6}$. The Laplacians of the $M$ - $\mathrm{B}$ bonds are $1.51 e / \AA^{5}$ for $\mathrm{LaB}_{6}$ and $1.33 \mathrm{e} / \AA^{5}$ for $\mathrm{BaB}_{6}$. It is still difficult to interpret the bond between heavy elements ${ }^{32}$. In the present charge density, several parameters cannot be refined for the heavy element. We do not describe the evaluation of the $M$ - $B$ bonds in the present paper.

The atomic basins of $\mathrm{La}$ and $\mathrm{B}$ atoms for $\mathrm{LaB}_{6}$ and $\mathrm{Ba}$ and $\mathrm{B}$ atoms for $\mathrm{BaB}_{6}$ are shown in Supplementary Figures $\mathrm{S} 5 \mathrm{a}$ and $\mathrm{S} 5 \mathrm{~b}$. The shape of atomic basin for $\mathrm{La}$ atom in $\mathrm{LaB}_{6}$ is very similar to that for $\mathrm{Ba}$ atom in $\mathrm{BaB}_{6}$. The triangle shape of atomic basin for $\mathrm{B}$ atom in $\mathrm{LaB}_{6}$ is almost identical to that in $\mathrm{BaB}_{6}$. The charges in atomic basins for $\mathrm{La}$ and $\mathrm{Ba}$ atoms were $55.17 e$ and $54.86 e$, which are corresponding to $+1.83 e$ and $+1.14 e$, respectively. The charges of $\mathrm{B}$ atoms were $-0.28 e$ for $\mathrm{LaB}_{6}$ and $-0.14 e$ for $\mathrm{BaB}_{6}$, respectively. It is found that the $\mathrm{B}_{6}$ cluster in $\mathrm{LaB}_{6}$ has approximately 0.7 more electrons than that in $\mathrm{BaB}_{6}$.

We successfully carried out multipole modelling of charge density connecting with topological analysis with sufficiently small reliability factors. In order to confirm the accuracy of structure factors and charge densities more precisely, we did the MEM charge density analysis of the present data. The charge densities of $\mathrm{LaB}_{6}$ and $\mathrm{BaB}_{6}$ using the observed structure factors from final powder fittings were calculated by the MEM. Figure $3 a$ and $b$ show the MEM charge densities of $\mathrm{LaB}_{6}$ and $\mathrm{BaB}_{6}$ for 020 plane as a contour map. The charge density at the $\mathrm{B}_{6}-\mathrm{B}_{6}$ bond midpoint is $1.06 e / \AA^{3}$ for $\mathrm{LaB}_{6}$ and $1.03 e / \AA^{3}$ for $\mathrm{BaB}_{6}$ which are much higher than those at $\mathrm{B}-\mathrm{B}$ bond midpoint, $0.69 e / \AA^{3}$ for $\mathrm{LaB}_{6}$ and $0.69 e / \AA^{3}$ for $\mathrm{BaB}_{6}$.

In order to perform quantitative comparison between the MEM charge densities and theoretical valence density, we developed the method for determination of an experimental valence charge density by the combination of MEM and multipole refinement. The experimental valence charge densities were calculated by the subtraction of core charge densities from the total MEM charge densities. The core charge densities were calculated by MEM using $F_{\text {core }}(h k l)$ which were the structure factors of core with thermal motion calculated by the XD program and the uncertainties of observed structure factors, $\sigma(h k l)$. Figure $3 \mathrm{c}$ and $\mathrm{d}$ show the valence charge densities for $\mathrm{LaB}_{6}$ and $\mathrm{BaB}_{6}$. The charge densities at the $\mathrm{B}_{6}-\mathrm{B}_{6}$ and $\mathrm{B}-\mathrm{B}$ bond midpoints were $1.06 e / \AA^{3}$ and $0.69 e / \AA^{3}$ for $\mathrm{LaB}_{6}$ and $1.03 e / \AA^{3}$ and $0.69 e / \AA^{3}$ for $\mathrm{BaB}_{6}$. The charge densities at the midpoint of $\mathrm{B}_{6}-\mathrm{B}_{6}$ bond are much larger than that at $\mathrm{B}-\mathrm{B}$ bond in both the materials. These features are consistent with the valence densities by multipole refinement and theoretical calculation. These results confirm the quality of structure factors in the present study is enough for accurate charge density studies.

The valence, deformation, and total charge densities of $\mathrm{LaB}_{6}$ are very similar to those of $\mathrm{BaB}_{6}$. The main features are the strong $\mathrm{B}_{6}-\mathrm{B}_{6}$ bond, $\mathrm{B}-\mathrm{B}$ bond and weak $M-\mathrm{B}$ bond in both the materials. To detect small difference between $\mathrm{LaB}_{6}$ and $\mathrm{BaB}_{6}$, we calculated a difference of valence charge densities between $\mathrm{LaB}_{6}$ and $\mathrm{BaB}_{6}$. The unit cells of both the materials were divided by $85 \times 85 \times 85$ pixels for charge density subtraction. The size of both the densities was normalized by the unit cell. Figure 4 shows the difference density as a contour map for 020 section obtained from the multipole valence densities. There are weak charge density peaks around $\mathrm{B}_{6}-\mathrm{B}_{6}$ bond whereas there are weak charge densities on the $\mathrm{B}_{6}-\mathrm{B}_{6}$ line. These facts indicate the charges along $\mathrm{B}_{6}-\mathrm{B}_{6}$ line are almost identical between $\mathrm{LaB}_{6}$ and $\mathrm{BaB}_{6}$. It is also clearly recognized that there are excess electrons in the center of $\mathrm{B}_{6}$ octahedra. 

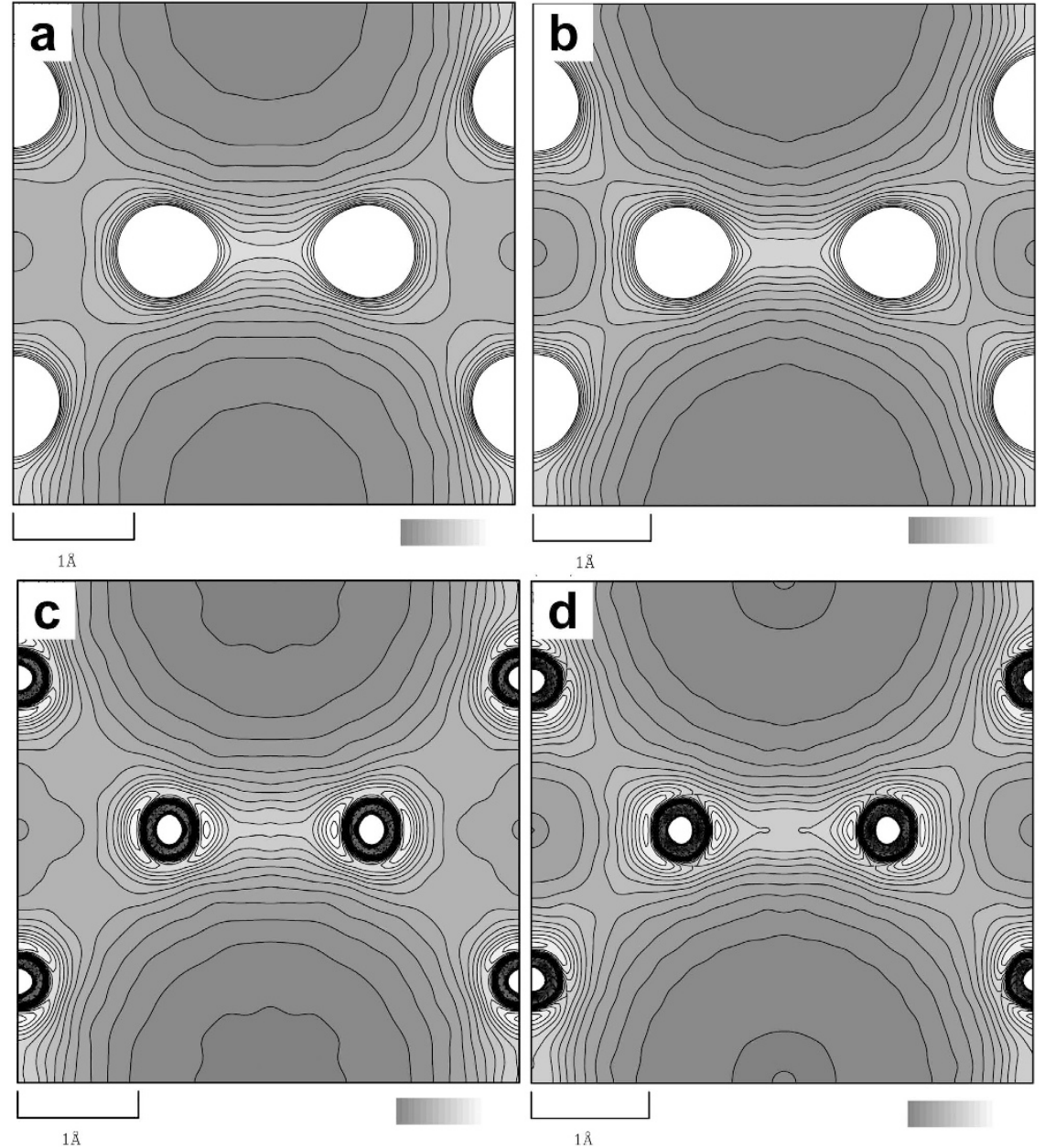

$1 \AA$

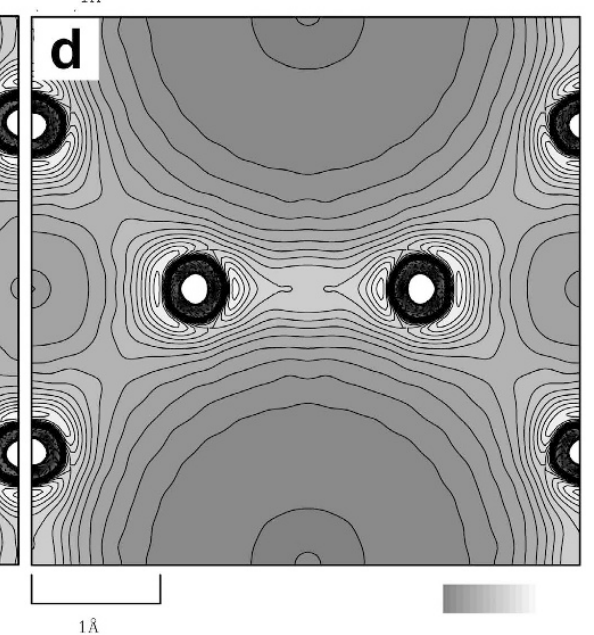

Figure 3. Charge densities of $\mathrm{LaB}_{6}$ and $\mathrm{BaB}_{6}$ based on the MEM. (a,b) Total charge density maps of $(\mathbf{a}) \mathrm{LaB}_{6}$ and (b) $\mathrm{BaB}_{6}$ for 020 plane. (c,d) Valence charge densities of (c) $\mathrm{LaB}_{6}$ and (d) $\mathrm{BaB}_{6}$ by the combination of the MEM and multipole refinement. Contour lines are drawn from 0.0 to 1.5 with $0.1 \mathrm{e} / \AA^{3}$ step widths. The maps are drawn by the program using pgplot library (http://www.astro.caltech.edu/ tjp/pgplot/) and edited with Gimp 2.8.14 (https://www.gimp.org).

\section{Discussion}

We have successfully detected the small difference of valence charges between $\mathrm{LaB}_{6}$ and $\mathrm{BaB}_{6}$. Two kinds of electron localized parts were found in the metallic $\mathrm{LaB}_{6}$. We calculated theoretical charge density of $\mathrm{LaB}_{6}$ using computer program WIEN $2 \mathrm{k}^{33}$ to investigate the observed charges. A theoretical band structure of $\mathrm{LaB}_{6}$ calculated by the present study is shown in Fig. $4 \mathrm{~b}$. This is consistent with the many previous studies ${ }^{9-12}$. Then we calculated and visualized charge densities by changing the energy level from -1.36 to $0 \mathrm{eV}$, from -2.72 to $-1.36 \mathrm{eV}$, and from -4.08 to $-2.72 \mathrm{eV}$ (see Supplementary Figures S6). The contour map with the energy level from -1.36 to $0 \mathrm{eV}$ is also shown in Fig. $4 \mathrm{~b}$. The charge density localization along with $\mathrm{B}_{6}-\mathrm{B}_{6}$ bond is recognized in Fig. $4 \mathrm{~b}$. We found the weak peaks along with $\mathrm{B}_{6}-\mathrm{B}_{6}$ line are consistent with the electron distributions just below the Fermi level in the theoretical charge density. The band structure of $\mathrm{BaB}_{6}$ has been reported by several researchers ${ }^{13,14}$. The energy bands from -2.72 to $-1.36 \mathrm{eV}$ and -4.08 to $-2.72 \mathrm{eV}$ of $\mathrm{LaB}_{6}$ also similarly exist in the band structure below Fermi level of $\mathrm{BaB}_{6}$. The band from -1.36 to $0.0 \mathrm{eV}$ of $\mathrm{LaB}_{6}$ does not exist in the reported band structure of $\mathrm{BaB}_{6}$. These facts confirm that the present study visualized the spatial distribution of electrons just below the Fermi level experimentally.

The charge localization in the center of $\mathrm{B}_{6}$ has never been found in the theoretical charge densities of Fig. $4 \mathrm{~b}$, S6a and S6b. The volume of $\mathrm{B}_{6}$ octahedron for $\mathrm{LaB}_{6}, 2.582 \AA^{3}$, is $2.5 \%$ smaller than that of $\mathrm{BaB}_{6}, 2.647 \AA^{3}$, from the present determined structure. Both the materials have bonding octahedron orbitals, such as $a_{1 \mathrm{~g}}{ }^{34}$. The electron density of these orbitals for $\mathrm{LaB}_{6}$ inside the octahedron should be higher than that of $\mathrm{BaB}_{6}$ due to the smaller volume. We calculated amount of the electrons observed at the center of $\mathrm{B}_{6}$ octahedron. The number of pixels in positive charge region inside $B_{6}$ octahedron is 8000 which is corresponding to $\left(a / 3^{*} \sqrt{ } 2\right)^{3}$. The electron density at the center of the octahedron is $0.13 e \AA^{-3}$. The total number of the electron inside the octahedron is $0.06 e$ which is $3 \%$ of two electron filled $a_{1 \mathrm{~g}}$ orbital. Therefore, the charge density in the $\mathrm{B}_{6}$ octahedron is mainly due to the volume difference. 


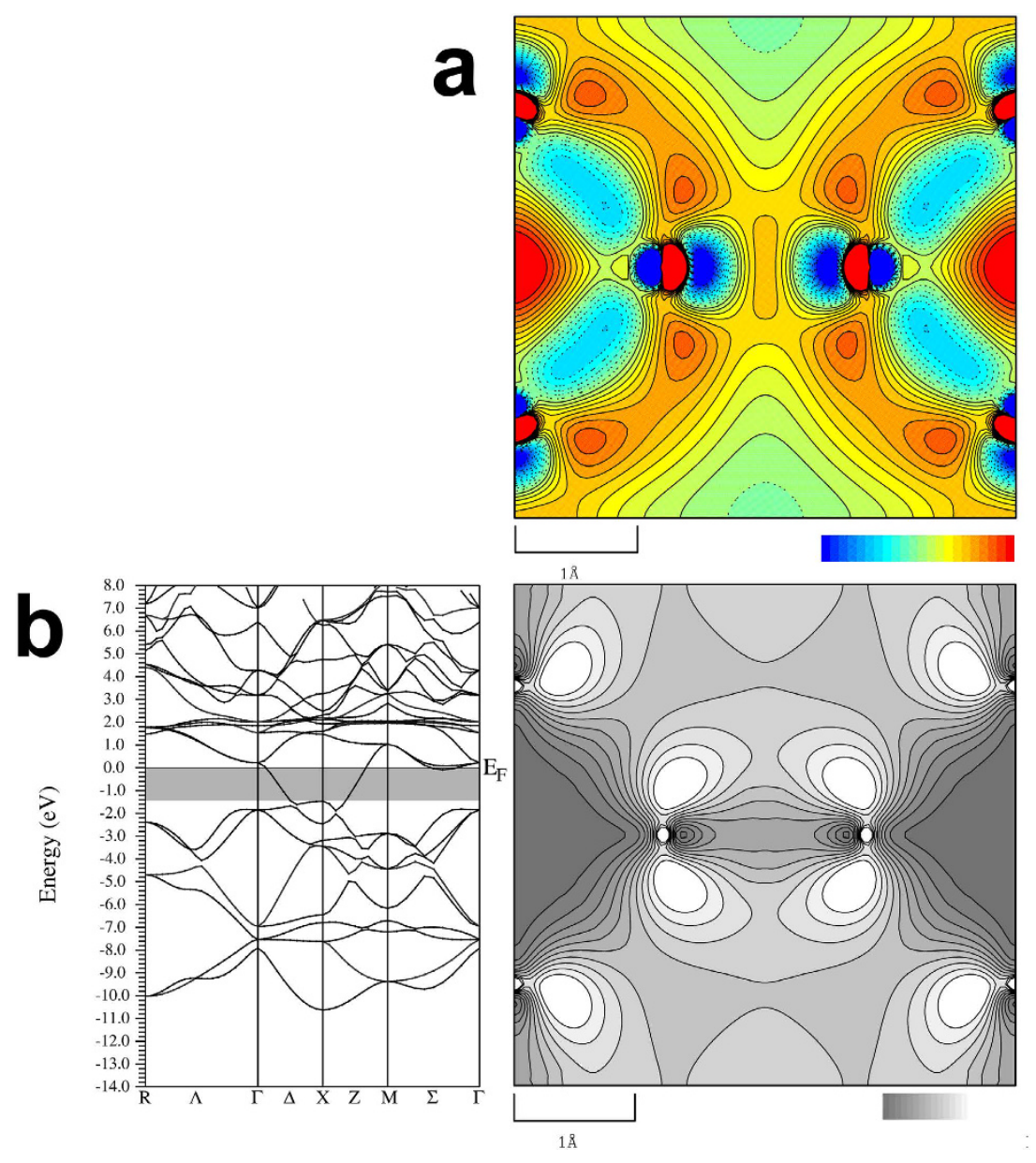

Figure 4. Contour map of difference of valence charge densities between $\mathrm{LaB}_{6}$ and $\mathrm{BaB}_{6}$ and $\mathrm{Band}_{\mathrm{and}}$ structure plot with contour map of the theoretical electron density with energy from -1.34 to $0.0 \mathrm{eV}$. (a) Difference of valence charge density between $\mathrm{LaB}_{6}$ and $\mathrm{BaB}_{6}$ based on multipole refinement. Contour lines are drawn from -0.1 to 0.1 with $0.01 e / \AA^{3}$ step widths. (b), Spin-up charge density with the band structure. The gray colored part in the band structure shows the energy of charge densities. Contour lines are drawn from 0.0 to 0.003 with $0.0003 \mathrm{e} / \AA^{3}$ step widths. The map is drawn by the program using pgplot library (http://www.astro. caltech.edu/ tjp/pgplot/) and edited with Gimp 2.8.14 (https://www.gimp.org).

In this paper, we have successfully observed the three-dimensional charge density distribution directly on and just beneath Fermi level by the X-ray charge density study. Quantitatively comparison between iso-structural solids with different electrical properties plays a crucial role for the detection. Most of the materials with exotic physical properties such as superconductor have the iso-structural and different property solids. The present experimental and analytical technique can be used for such system. Electron density distribution is now one of the most information-rich observable owing to the great improvement of experimental situation such as synchrotron X-ray source.

\section{Methods}

Powder sample preparation. The powder samples of $3 \mathrm{~N}$ purity $\mathrm{LaB}_{6}$ with less than 350 mesh particle size and $2 \mathrm{~N} 5$ purity $\mathrm{BaB}_{6}$ with less than 100 mesh particle size were purchased from Mitsuwa Chemicals Co., Ltd. The samples were ground using an $\mathrm{Al}_{2} \mathrm{O}_{3}$ mortar. The fine particles with less than $5 \mu \mathrm{m}$ size were selected by the precipitation method with ethanol as a solvent. The selected fine particles were agglomerated together with tiny amounts of glue. The samples were cut into a rectangle. By using these samples, we did not need to use glass capillary in the experiment. This is effective to reduce background scattering in diffraction data. The photographs of samples are shown in the insets of Fig. $1 \mathrm{a}$ and $\mathrm{b}$. The size of the samples were $3.5 \mathrm{~mm} \times 0.25 \mathrm{~mm} \times 0.27 \mathrm{~mm}$ for $\mathrm{LaB}_{6}$ and $3.5 \mathrm{~mm} \times 0.23 \mathrm{~mm} \times 0.29 \mathrm{~mm}$ for $\mathrm{BaB}_{6}$ as shown in the Figures.

Synchrotron X-ray powder diffraction measurement. The powder diffraction data were measured by a Large Debye-Scherrer camera with an Imaging Plate (IP) as a detector at BL02B2 beamline ${ }^{16}$. Wavelength

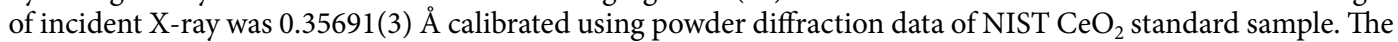
high-energy X-ray was used for reducing an effect of absorption. In the case of $\mathrm{LaB}_{6}$, the angular dependence of absorption between $0^{\circ}$ and $120^{\circ}$ at $2 \theta$ is less than $0.9 \%$. The $120^{\circ}$ at $2 \theta$ is the maximum diffraction angle in the present study. In the case of $\mathrm{BaB}_{6}$, the dependence is less than $0.7 \%$. The collimator size was $3.0 \times 0.5 \mathrm{~mm}$. 
We measured two datasets using an overlaid measurement technique ${ }^{24}$ for $\mathrm{LaB}_{6}$ and $\mathrm{BaB}_{6}$. One of the data was measured by moving IP cassette to measure high order data, D2. The D2 data were measured by moving IP with long exposure time, 80 minutes for $\mathrm{LaB}_{6}$ and 120 minutes for $\mathrm{BaB}_{6}$. We recognized Bragg peaks at better than $d>0.22 \AA$ A resolution range in the D2 data. Another data, D1, using a normal procedure which includes the high intense low-angle Bragg reflections were measured with 20 minutes and 30 minutes exposure times for $\mathrm{LaB}_{6}$ and $\mathrm{BaB}_{6}$, respectively. Temperatures of samples for all the measurement were controlled at $100 \mathrm{~K}$ using $\mathrm{N}_{2}$ gas flow devices.

Data analysis using relativistic atomic scattering factors. An analysis of ultra-high resolution data requires an external treatment. It is impossible to analyze the data by using atomic scattering factors expressed by four terms Gaussian which are listed in International Table B and used in normal crystallography software. Since the equation cannot represent high order data with $d<0.25 \AA$, we used relativistic atomic scattering factors calculated by $\mathrm{Su}$ and Coppens ${ }^{35}$. By using the scattering factors, powder fittings in high order region were drastically improved. The present compounds include relatively heavier elements. The fittings in high angle regions in the present study represent requirements and abilities of relativistic scattering factors from experiment.

The $2 \theta$ ranges of Rietveld analysis for $\mathrm{LaB}_{6}$ were from $1.9^{\circ}$ to $67.5^{\circ}$ for $\mathrm{D} 1$ and from $54.8^{\circ}$ to $118.35^{\circ}$ for $\mathrm{D} 2$. The ranges for $\mathrm{BaB}_{6}$ were from $1.9^{\circ}$ to $67.37^{\circ}$ for $\mathrm{D} 1$ and from $52.5^{\circ}$ to $105.055^{\circ}$ for $\mathrm{D} 2$. Reliability factors based on weighted profile, $R_{\mathrm{wp}}$, and Bragg intensity, $R_{\mathrm{I}}$, of $\mathrm{LaB}_{6}$ were $6.50 \%$ and $1.13 \%$ for $\mathrm{D} 1,0.75 \%$ and $0.87 \%$ for $\mathrm{D} 2$, and $2.27 \%$ and $0.97 \%$ for two datasets and those of $\mathrm{BaB}_{6}$ were $5.97 \%$ and $1.09 \%$ for $\mathrm{D} 1,0.88 \%$ and $1.28 \%$ for $\mathrm{D} 2$, and $2.22 \%$ and $1.22 \%$ for two datasets.

Multipole refinement. The $[1 s]^{2}$ were core and $[2 s 2 p]^{3}$ were valence electrons for boron atom for the refinement. The $[\mathrm{Xe}]^{54}$ were core and $[6 \mathrm{~s} 5 \mathrm{~d}]^{3}$ were valence for La atom. The $[\mathrm{Xe}]^{54}$ were core and $[6 \mathrm{~s}]^{2}$ were valence for $\mathrm{Ba}$ atom. Local coordinates for $\mathrm{B}$ and $M(=\mathrm{La}, \mathrm{Ba})$ atoms were defined for the multipole refinement. The $x-, y$-, and $z$ - axes of the local coordinate for metal atoms were parallel to [100], [010], and [001] directions. The $x$-, $y$-, and $z$ - axes for $\mathrm{B}$ with $4 \mathrm{~mm}$ site symmetry were $[x, 1 / 2,1],[x, 1,1 / 2]$ and $[-1,1 / 2,1 / 2]$ directions. The multipole populations which can be refined for $M$ site are $P_{40}$ and $P_{44+}$ where the $P_{44+}$ is $0.74045 P_{40}$ from the symmetry restriction. The populations which can be refined for B site are $P_{10}, P_{20}, P_{30}, P_{40}$, and $P_{44+}$.

Charge density study by Maximum Entropy Method. The unit cells of $\mathrm{LaB}_{6}$ and $\mathrm{BaB}_{6}$ were divided by $128 \times 128 \times 128$ pixels. The MEM analyses were carried out using computer program ENIGMA ${ }^{36}$. Total numbers of Bragg reflections were 898 for $\mathrm{LaB}_{6}$ and 775 for $\mathrm{BaB}_{6}$. Reliability factors of MEM analysis were $R=0.47 \%$ and $R_{\mathrm{W}}=0.53 \%$ for $\mathrm{LaB}_{6}$ and $R=0.58 \%$ and $R_{\mathrm{W}}=0.47 \%$ for $\mathrm{BaB}_{6}$.

Theoretical calculation by WIEN2k. Theoretical band structure and charge density of $\mathrm{LaB}_{6}$ were calculated using full potential-linearized augmented plane wave (FP-LAPW) method with the generalized gradient approximation (GGA) by WIEN2k package ${ }^{33}$. The calculation was performed using structure information including lattice constants and atomic coordinates from the result of the present Rietveld refinement. We used $1000 \mathrm{k}$ points with $R^{\mathrm{MT}} K_{\max }=7.0$, where $R^{\mathrm{MT}}$ is the Muffin-Tin radius and $K_{\max }$ is the maximum value of the wave vector, in the calculation.

\section{References}

1. Bednorz, J. G. \& Müller, K. A. Possible high $T_{\mathrm{c}}$ superconductivity in the Ba-La-Cu-O system. Z. Phys. B 64, 189-193 (1986).

2. Schell, G., Winter, H., Rietschel, H. \& Gompf, F. Electronic structure and superconductivity in metal hexaborides. Phys. Rev. B 25, 1589-1599 (1982).

3. Etourneau, J. \& Hagenmuller, P. Structure and physical features of the rare-earth borides. Philos. Mag. B 52, 589-610 (1985)

4. Herzig, P. et al. Electric-field-gradient tensor and charge densities in $\mathrm{LaB}_{6}:{ }^{11} \mathrm{~B}$ nuclear-magnetic-resonance single-crystal investigations and first-principles calculations. J. Appl. Phys. 103, 083534 (2008).

5. Gesley, M. \& Swanson, L. W. A determination of the low work function plates of LaB 6 . Surf. Sci. 146, 583-599 (1984).

6. Xiao, L. et al. Origins of high visible light transparency and solar heat-shielding performance in LaB L Appl. Phys. Lett. 101, 041913 (2012).

7. Arko, A. J., Crabtree, G., Karim, D., Mueller, F. M. \& Windmiller, L. R. De Haas-van Alphen effect and the Fermi surface of LaB 6 . Phys. Rev. B 13 5240-5247 (1976).

8. Kontrym-Sznajd, G., Samsel-Czekala, M., Biasini, M. \& Kubo, Y. Band structure of LaB $_{6}$ by an algorithm for filtering reconstructed electron-positron momentum densities. Phys. Rev. B 70, 125103 (2004).

9. Hossain, F. M., Riley, D. P. \& Murch, G. E. Ab initio calculations of the electronic structure and bonding characteristics of LaB 6 . Phys. Rev. B 72, 235101 (2005).

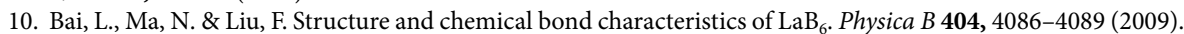

11. Alarco, J. A., Talbot, P. C. \& Mackinnon, I. D. R. Comparison of Functionals for Metal Hexaboride Band Structure Calculations. Modeling and Numerical Simulation of Material Science 4, 53-69 (2014).

12. Luo, K. et al. Crystal structures and mechanical properties of $\mathrm{M}(\mathrm{Mg}, \mathrm{Sr}, \mathrm{Ba}, \mathrm{La})_{\mathrm{X}} \mathrm{Ca}_{1-\mathrm{x}} \mathrm{B}_{6}$ solid solution: A first principles study. Ceram. Int. 42, 6632-6639 (2016).

13. Huang, B., Duan, Y.-H., Sun, Y., Peng, M.-J. \& Chen, S. Electronic structures, mechanical and thermodynamic properities of cubic alkaline-earth hexaborides from first principles calculations. J. Alloys Compds. 635, 213-224 (2015).

14. Schmitt, K., Stückl, C., Ripplinger, H. \& Albert, B. Crystal and electronic structure of $\mathrm{BaB}_{6}$ in comparison with $\mathrm{CaB}_{6}$ and $\mathrm{molecular}$ $\left[\mathrm{B}_{6} \mathrm{H}_{6}\right]^{2-}$. Solid State Sci. 3, 321-327 (2001).

15. Massidda, S., Monnier, R. \& Stoll, E. Electronic Structure of barium hexaboride. Eur. Phys. J. B 17, 645-649 (2000).

16. Nishibori, E. et al. The large Debye-Scherrer camera installed at SPring-8 BL02B2 for charge density studies. Nucl. Instr. Meth. Phys. Res. A 467, 1045-1048 (2001).

17. Nishibori, E., Shibata, T., Kobayashi, W. \& Moritomo, Y. Bonding nature of $\mathrm{LiCoO}_{2}$ by topological analysis of electron density from X-ray diffraction. Electrochemistry 83, 840-842 (2015).

18. Sakata, M. \& Sato, M. Accurate structure analysis by the maximum-entropy method. Acta Crystallogr. A 46, 263-270 (1990).

19. Hansen, N. K. \& Coppens, P. Testing aspherical atom refinements on small-molecule data sets. Acta Crystallogr. A 34, 909-921 (1978). 
20. Nishibori, E., Hyodo, H., Kimura, K. \& Takata, M. Revisit: High resolution charge density study of $\alpha$-rhombohedral boron using third-generation SR data at SPring-8. Solid State Sci. 47, 27-31 (2015).

21. Kasai, H. \& Nishibori, E. Multiple powder diffraction data for an accurate charge density study using synchrotron radiation X-ray. Phys. Scripta 91, 043009 (2016).

22. Ohno, A. et al. X-ray charge density study of chemical bonding in skutterudite $\mathrm{CoSb}_{3}$. Phys. Rev. B 76, 064119 (2007).

23. Schmøkel, M. S. et al. Comparative study of X-ray charge-density data on $\mathrm{CoSb}_{3}$. Acta Crystallogr. A 69, 570-582 (2013).

24. Nishibori, E. et al. Accurate structure factors and experimental charge densities from synchrotron X-ray powder diffraction data at SPring-8. Acta Crystallogr. A 63, 43-52 (2007).

25. Svendsen, H. et al. Multipole electron-density modelling of synchrotron powder diffraction data: the case of diamond. Acta Crystallogr. A 66, 458-469 (2010).

26. Bindzus, N. et al. Experimental determination of core electron deformation in diamond. Acta Crytallogr. A70, 39-48 (2014).

27. Wahlberg, N. et al. Synchrotron powder diffraction of silicon: high-quality structure factors and electron density. Acta Crystallogr. A72, 28-35 (2016).

28. Wahlberg, N. et al. Powder X-ray Diffraction Electron Density of Cubic Boron Nitride. J. Phys. Chem. C. 119, 6164-6173 (2015).

29. Wahlberg, N. et al. Low-temperature powder X-ray diffraction measurements in vacuum: analysis of the thermal displacement of copper. J. Appl. Crystallogr. 49, 110-119 (2016).

30. Volkov, A. et al. XD2006. A Computer Program Package for Multipole Refinement, Topological Analysis of Charge Densities and Evaluation of Intermolecular Energies from Experimental or Theoretical Structure Factors. University at Buffalo, State University of New York, NY, USA; University of Milano, Italy; University of Glasgow, UK; CNR-ISTM, Milano, Italy; Middle Tennessee State University, TN, USA (2006).

31. Chen, C. H., Aizawa, T., Iyi, N., Sato, A. \& Otani, S. Structural refinement and thermal expansion of hexaborides. J. Alloys. Comp. 366, L6-L8 (2004).

32. Macchi, P. \& Sironi, A. Chemical bonding in transition metal carbonyl clusters: complementary analysis of theoretical and experimental electron densities. Cood. Chem. Rev. 239-239, 383-412 (2003).

33. Blaha, P., Schwarz, K., Madsen, G. K. H., Kvasnicka, D. \& Luitz, J. WIEN2K, an Augmented Plane Wave + Local Orbitals Program for Calculating Crystal Properties. Karlheinz Schwarz Technische Universitat, Wien, Austria (2001).

34. Longuet-Higgins, H. C. \& Roverts, M. de, V. The electronic Structure of the Borides MB $_{6}$. Proc. Roy. Soc. Lond. A 224, 336-347 (1954).

35. Su, Z. \& Coppens, P. Relativistic $\mathrm{x}$-ray elastic scattering factors for neutral atoms $\mathrm{Z}=1-54$ from multiconfiguration Dirac-Fock wavefunctions in the $0-12 \AA^{-1} \sin \theta / \lambda$ range, and six-Gaussian analytical expressions in the $0-6 \AA^{-1}$ range. Acta Crystallogr. A 53, 749-762 (1997).

36. Tanaka, H. et al. ENIGMA: maximum-entropy method program package for huge systems. J. Appl. Crystallogr. 35, 282-286 (2002).

37. Ozawa, T. C. \& Kang. Sung, J. Balls \& Sticks: Easy-to-Use Structure Visualization and Animation Creating Program. J. Appl. Crystallogr. 37, 679 (2004).

\section{Acknowledgements}

We thank Mr. A. Kurita for his experimental and analytical help. This work has been partly supported by JSPS KAKENHI Grant Numbers 16K13660, 25600148, and 20360006. This work also has been partly supported by Futaba Electronics Memorial Foundation and the Naito Foundation. The synchrotron radiation experiments were performed at SPring-8 with the approval of the Japan Synchrotron Radiation Research Institute (JASRI) as a Power User (Proposal No: 2006A0096 and 2009A0084) and a Partner User (Proposal No: 2015A0074).

\section{Author Contributions}

E.N. was responsible for designing and coordinating this study. E.N. carried out SR-PXRD measurements. H.K. and E.N. interpreted and discussed the results and structural analysis. E.N. calculated and analyzed charge densities. H.K. and E.N. were involved in discussions. H.K. and E.N. commented on the paper, which was written by H.K. and E.N.

\section{Additional Information}

Supplementary information accompanies this paper at http://www.nature.com/srep

Competing financial interests: The authors declare no competing financial interests.

How to cite this article: Kasai, H. and Nishibori, E. Spatial distribution of electrons near the Fermi level in the metallic $\mathrm{LaB}_{6}$ through accurate X-ray charge density study. Sci. Rep. 7, 41375; doi: 10.1038/srep41375 (2017).

Publisher's note: Springer Nature remains neutral with regard to jurisdictional claims in published maps and institutional affiliations.

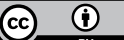

This work is licensed under a Creative Commons Attribution 4.0 International License. The images or other third party material in this article are included in the article's Creative Commons license, unless indicated otherwise in the credit line; if the material is not included under the Creative Commons license, users will need to obtain permission from the license holder to reproduce the material. To view a copy of this license, visit http://creativecommons.org/licenses/by/4.0/

(c) The Author(s) 2017 